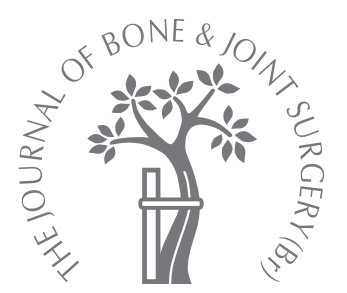

S. W. Veitch, S. L. Whitehouse, J. R. Howell, M. J. W. Hubble, G. A. Gie, A. J. Timperley

From the Princess Elizabeth

Orthopaedic Centre, Royal Devon and Exeter Hospital, Exeter, United Kingdom

S. W. Veitch, MD, FRCS(Tr \& Orth), Consultant Orthopaedic Surgeon

Salisbury District Hospital, Odstock Road, Salisbury, Wiltshire SP2 8BJ, UK.

S. L. Whitehouse, PhD Research Fellow, Biostatistician Orthopaedic Research Unit, Institute of Health and Biomedical Innovation Queensland University of Technology, The Prince Charles Hospital, Brisbane, Queensland, Australia.

In J. R Howell, FRCS(Trauma\&Orth), Consultant Orthopaedic Surgeon M. J. W. Hubble, FRCS(Trauma\&Orth), Consultant Orthopaedic Surgeon

G. A. Gie, MBChB, FRCS(Ed), Consultant Orthopaedic Surgeon A. J. Timperley, DPhil(Oxon), Consultant Orthopaedic Surgeon Exeter Hip Unit

Princess Elizabeth Orthopaedic Centre, Royal Devon \& Exeter Hospital, Barrack Road, Exeter EX2 5DW, UK.

Correspondence should be sent to Mr S. W. Veitch; e-mail: swveitch@doctors.org.uk

(C)2010 British Editorial Society of Bone and Joint Surgery doi:10.1302/0301-620X.92B10. $24054 \$ 2.00$

$J$ Bone Joint Surg [Br] 2010;92-B:1351-5.

Received 26 November 2010; Accepted after revision 18 May 2010

\title{
The concentric all-polyethylene Exeter acetabular component in primary total hip replacement
}

\begin{abstract}
We report the outcome of the flangeless, cemented all-polyethylene Exeter acetabular component at a mean of 14.6 years (10 to 17) after operation. Of the 263 hips in 243 patients, 122 prostheses are still in situ; 112 patients (119 hips) have died, 18 hips have been revised, and three patients (four hips) were lost to follow-up. Radiographs at the final review were available for 110 of the 122 surviving hips. There were acetabular radiolucent lines in $\mathbf{5 4}$ hips (49\%). Two acetabular components had migrated but neither patient required revision. The Kaplan-Meier survivorship at $\mathbf{1 5}$ years with $\mathbf{6 1}$ hips at risk with revision for any cause as the endpoint was $89.9 \%$ (95\% confidence interval (Cl) 84.6 to 95.2 ) and for aseptic loosening of the acetabular component or lysis $91.7 \%$ (95\% Cl 86.6 to 96.8). In 210 hips with a diagnosis of primary osteoarthritis, survivorship with revision for any cause as the endpoint was 93.2\% (95\% Cl 88.1 to 98.3), and for aseptic loosening of the acetabular component $95.0 \%$ (95\% Cl 90.3 to 99.7$)$.

The cemented all-polyethylene Exeter acetabular component has an excellent long-term survivorship.
\end{abstract}

Both cemented metal-backed and all-polyethylene acetabular components were used at our institution between 1984 and 1991. Metalbacked devices were abandoned when it became apparent that the metal-backing was responsible for polyethylene and cement debris. ${ }^{1}$ Despite this, a review of the first 325 Exeter Universal hips (Stryker, Newbury, United Kingdom) of which $94 \%$ had metalbacked acetabular components, described $96.9 \%$ (95\% confidence interval (CI) 93.1 to 89.9) survivorship with revision for aseptic loosening of the acetabular component as the endpoint at 12 years $^{2}$ and $90.4 \%(95 \%$ CI 83.1 to 94.7$)$ at 17 years. $^{3}$ Since 1991, allpolyethylene cemented components have been implanted. The long-term performance of these has not previously been fully established.

Two designs of all-polyethylene component were used, with different polyethylene thicknesses at the dome, described as low or high profile, in which the high profile design permits greater lateralisation of the femoral head. They had a skirt on the external face designed to reduce the possibility of dislocation and had no added flange (Fig. 1). We describe the survivorship and the clinical and radiological outcomes of these allpolyethylene implants.

\section{Patients and Methods}

We reviewed a consecutive group of patients operated on between March 1991 and December 1993, who underwent primary total hip replacement (THR) using the Exeter all-polyethylene acetabular component and the Exeter Universal femoral component (Stryker). Patients who had undergone previous hip surgery were excluded. Clinical data were prospectively acquired and stored on a database.

A total of 263 primary THRs in 243 patients were included, of which 107 were in men and 156 in women. The mean age of the patients at the time of operation was 66.3 years (18 to 89 ); 25 patients were $<50$ years old. Consultant orthopaedic surgeons carried out $64 \%$ of the operations, registrars and fellows $36 \%$. There were 210 hips $(80 \%)$ for which the initial diagnosis was osteoarthritis (Table I).

The posterior approach was used in 259 hips and the transgluteal (direct lateral) Hardinge approach ${ }^{4}$ in four hips. All implants were cemented. The femoral head diameter was $26 \mathrm{~mm}$ for 211 THRs $(80 \%)$ and the remainder had $30 \mathrm{~mm}$ diameter heads. Acetabular preparation involved removal of the surrounding osteophytes and decortication of the acetabular wall and roof wherever this was possible. A total of 35 hips (13\%) required augmentation of the acetabulum to address 


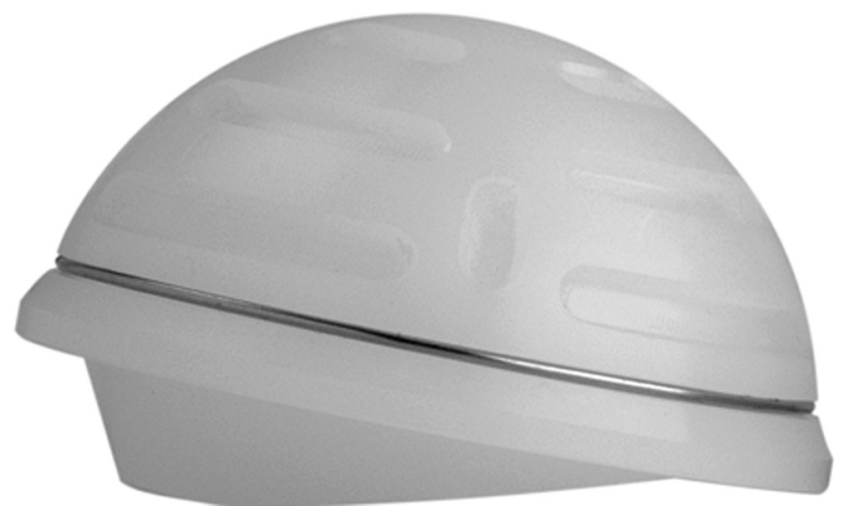

Fig. 1a

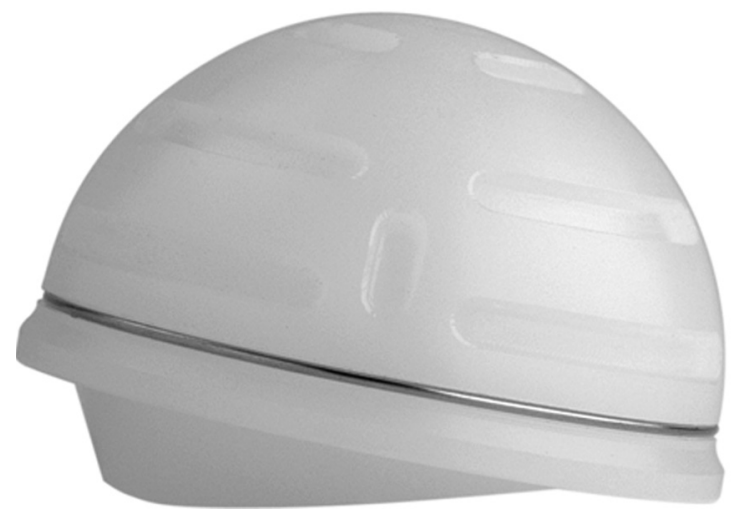

Fig. 1b

Drawing showing the low profile a) and high profile b) Exeter allpolyethylene acetabular component.

structural defects. This comprised block autografts in four hips $(2 \%)$, impaction grafting in 31 hips $(12 \%)$ and five hips $(2 \%)$ required both block and impaction grafting. In 13 hips $(5 \%)$ fixation in the form of plates or screws was required to support the graft. Multiple drill holes were made for cement fixation into host bone. The low profile version of the acetabular component was used in 232 hips $(88 \%)$ and the remaining 31 hips $(12 \%)$ received the high profile component. Simplex-cement (Stryker) was used in 204 hips (78\%), and Palacos cement (Heraeus Medical, Hanau, Germany) in 59 (22\%). All patients received Cefuroxime $1.5 \mathrm{~g}$ intravenously on induction of anaesthesia and two subsequent doses of $750 \mathrm{mg}$ at intervals of eight hours. Prophylaxis for deep-vein thrombosis included the wearing of anti-embolism stockings, and elevation of the foot in bed.

Clinical evaluation was by the grading system of Merle d'Aubigné and Postel as modified by Charnley, ${ }^{5}$ and at review using the Harris Hip score (HHS) ${ }^{6}$ and Oxford Hip score (OHS). ${ }^{7}$ The latter was used in the transformed 0 to 48 worst-to-best score as recommended by Murray et al. ${ }^{8}$ The Charnley categories (A, B or C) ${ }^{9}$ and the pre-operative
Table I. Pre-operative primary diagnosis

\begin{tabular}{lr}
\hline Primary diagnosis & Number (\%) \\
\hline Osteoarthritis & $210(80)$ \\
Dysplasia & $13(5)$ \\
Rheumatoid arthritis & $12(5)$ \\
Other inflammatory arthritis & $6(2)$ \\
Avascular necrosis & $8(3)$ \\
Post-traumatic osteoarthritis & $3(1)$ \\
Perthes' disease & $3(1)$ \\
Other & $8(3)$ \\
\hline
\end{tabular}

status of the patients are summarised in Table II. Patients were reviewed every five years following surgery.

Radiographs taken before and after operation and at the final the review were examined jointly by two surgeons (AJT, SWV) for evidence of fracture of the acetabular or femoral component, migration of the acetabular component (change in position on serial radiograph ${ }^{10}$ ), the location of radiolucent lines as defined by Kobayashi et $\mathrm{a}^{11}$ for the acetabular component according to DeLee and Charnley ${ }^{12}$ for the femoral stem according to Gruen, McNeice and Amstutz $^{13}$ at the cement-bone, cement-acetabular and cement-stem interfaces, the presence of localised lysis (bone osteolysis adjacent to the prosthesis) and measurements of linear wear using the Livermore technique. ${ }^{14}$

Survivorship analysis was performed using the KaplanMeier method ${ }^{10,15,16}$ for the entire cohort, as well as for the subset of patients with an initial diagnosis of osteoarthritis, with four different endpoints: re-operation for any reason; revision for aseptic loosening according to Hodgkinson, Shelley and Wroblewski ${ }^{17}$ or lysis surrounding the acetabular component, revision for aseptic loosening of the femoral component and the worst-case scenario ${ }^{18}$ with at least 40 cases remaining at risk. ${ }^{19,20}$

Statistical analysis. An analysis of the wear rates for components with and without radiolucent lines was conducted using Student's $t$-test with a p-value of 0.05 considered statistically significant.

\section{Results}

At the time of review 112 patients (119 hips) had died at a mean of 7.8 years (0.1 to 16) post-operatively, none of whom had undergone revision. One patient died three days following surgery due to a cerebral vascular haemorrhage. Three patients (four hips) were lost to follow-up. Of the remaining 128 patients (140 hips), 17 (18 hips) underwent revision at a mean of 11.0 years (3.2 to 15.9) post-operatively. Of these, 11 hips were revised for aseptic loosening of the acetabular component, three for recurrent dislocation, two for lysis around a well fixed implant and two for deep infection. All 13 hips revised for aseptic loosening of the acetabular component or lysis were symptomatic. The resulting acetabular defects according to the classification of Paprosky et $\mathrm{al}^{21}{ }^{21}$ were one type 1 , 


$\begin{aligned} & \text { Table II. Pre-operative (263 hips) and review (122 surviving hips) clinical scores using the Charnley } \\
& \text { modification of the Merle d'Aubigné and Postel scoring system (0 to } 6 \text { worst to best) }\end{aligned}$
\begin{tabular}{lcccc} 
Charnley category & Number of hips & $\%$ Pain & \% Function & $\%$ Movement \\
\hline $\begin{array}{l}\text { Pre-operative } \\
\text { A }\end{array}$ & 100 & 2.3 & 2.8 & 3.4 \\
B & 99 & 2.3 & 2.7 & 3.2 \\
C & 64 & 1.8 & 2.1 & 3.0 \\
& & & & \\
At review & & & & 5.6 \\
A & 29 & 5.6 & 5.6 & 5.4 \\
B & 57 & 5.5 & 5.0 & 5.6 \\
C & 36 & 5.5 & 3.7 & \\
\hline
\end{tabular}

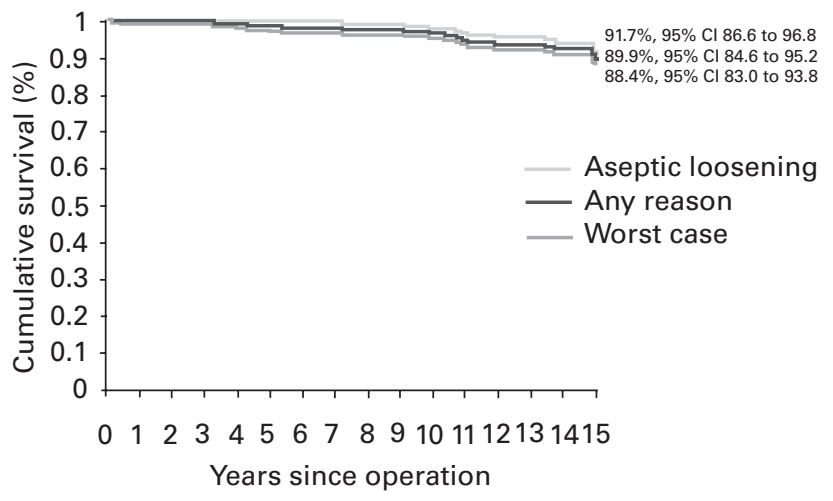

Fig. 2

Kaplan-Meier survival curves of the Exeter acetabular component $(95 \%$ confidence intervals $(\mathrm{CI})$ ) for all cases.

seven type $2 \mathrm{~A}$, three type $2 \mathrm{~B}$, one type $3 \mathrm{~A}$ and one type 3B. Revision to a cemented acetabular component was performed in five hips, and to an uncemented component in eight. Only two of the revisions required acetabular augmentation with rim mesh and impaction grafting. Of the 13 hips revised for aseptic loosening of the acetabular component, five had undergone acetabular grafting (two with block graft) at the time of initial surgery, one had a Paprosky type 1 acetabular defect, three had type $2 \mathrm{~A}$, and one had a type 3B defect. No hips required revision for aseptic loosening of the femoral component.

The Kaplan-Meier survivorship at 15 years with 61 hips remaining at risk, with revision for any cause as the endpoint was $89.9 \%$ (95\% CI 84.6 to 95.2 ) and for aseptic loosening of the acetabular component or lysis $91.7 \%$ (95\% CI 86.6 to 96.8 ). Worst-case survival at 15 years was $88.4 \%$ (95\% CI 83.0 to 93.8) (Fig. 2). In hips with a diagnosis of primary osteoarthritis (210 hips) survivorship with revision for any cause as the endpoint was 93.2\% (95\% CI 88.1 to 98.3) and for aseptic loosening of the acetabular component $95.0 \%$ (95\% CI 90.2 to 99.7) (Fig. 3).

A total of 113 patients (122 hips) who still had the prostheses in situ underwent review at a mean 14.6 years (10 to 17) post-operatively. Their clinical status at the last follow-

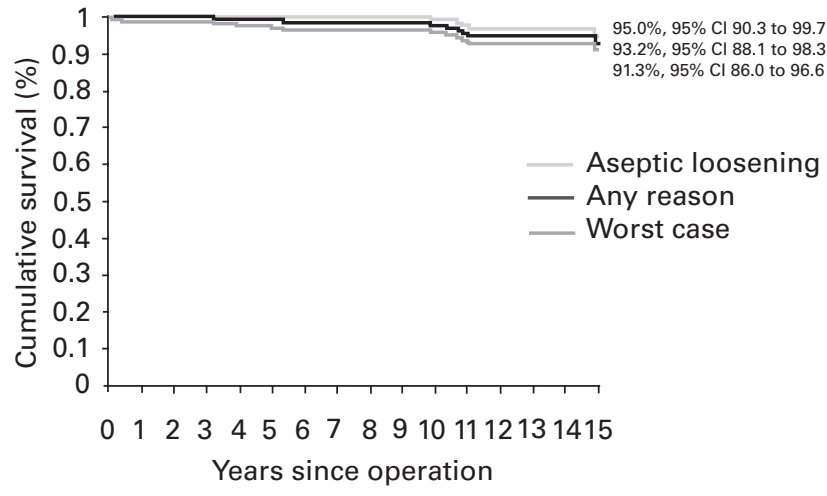

Fig. 3

Kaplan-Meier survival curves of the Exeter acetabular component $(95 \%$ confidence intervals $(\mathrm{CI})$ ) in patients with osteoarthritis.

up is shown in Table II. The mean OHS was 39 (7 to 48), and the mean HHS was 75 (21 to 91). In all, 16 patients were unable to attend for review and it was performed by telephone and radiographs were requested locally. The three patients (four hips) who were lost to follow-up were included as failures in the worst-case curve.

Complications. Deep-vein thrombosis occurred in three patients of whom one had a pulmonary embolism. A femoral nerve palsy occurred in one patient, and five hips dislocated, four late and one at six weeks post-operatively following a fall. One patient had cortical perforation of the tip of the femoral component during surgery. This did not affect the outcome. One patient sustained a periprosthetic fracture at the tip of the femoral component following a fall three years post-operatively. This was treated by open reduction and internal fixation using a plate and cables without disturbing the femoral component.

Initial post-operative anteroposterior and lateral radiographs were available for 101 hips and final follow-up radiographs for 110 of the 122 surviving hips. Radiolucent lines were found around the acetabular component in 22 hips with available radiographs $(22 \%)$ on the initial post-operative radiograph, 16 in DeLee and Charnley, zone 1 , one in zone 2 , two in zone 3 , one in zones 1 and 3 , and two in all three zones. Progression of radiolucent lines was 
seen in only one of these hips. At the latest follow-up, acetabular radiolucent lines were seen in 54 hips (49\%), 39 in zone 1 , two in zone 2 , two in zone 3 , three in zones 1 and 2, two in zones 1 and 3 , and $6(5 \%)$ in all three zones. A focal area of pelvic osteolysis was identified in five hips $(5 \%)$, four in zone 1 and one in both zones 1 and 2 . In two hips the acetabular component had migrated but the patients were asymptomatic and neither has been revised. Both of these components had radiolucencies but not all zones were affected. Applying the definition of radiological loosening of Hodgkinson et al, ${ }^{17}$ eight hips $(7 \%)$ were loose. Localised femoral osteolysis was seen in one hip in Gruen zone 5 and was not progressing. The mean linear wear of the polyethylene measured in 95 prostheses using the Livermore technique ${ }^{14}$ was $0.11 \mathrm{~mm} /$ year (0.0 to 0.4 ). No significant difference (Student's $t$-test; $\mathrm{p}=0.22$ ) was found in the linear wear in those components with no radiolucent lines, $0.10 \mathrm{~mm} /$ year (0 to 0.33 ), compared with those with one or more, $0.11 \mathrm{~mm} /$ year $(0.02$ to 0.37$)$. Of the 18 hips revised three acetabular components were high profile from 31 originally implanted and 15 low profile from 232 implanted.

\section{Discussion}

The survivorship at 15 years with revision for any cause as the endpoint was $89.9 \%$ (95\% CI 84.6 to 95.2 ) and in those with a diagnosis of primary osteoarthritis $93.2 \%$ (95\% CI 88.1 to 98.3 ). These results are comparable with those found in the Swedish hip registry where the ten-year survivorship with revision for any cause as the endpoint for 6374 cemented Exeter hips was $92.6 \%(95 \% \mathrm{CI} \pm 0.8 \%) .{ }^{22}$ At this time uncemented acetabular components had poorer results in both the Swedish and Norwegian registries..$^{21,23}$

The results of the all-polyethylene component are similar to the cemented metal-backed polyethylene implant previously reported from our unit. ${ }^{2,3}$

Radiolucent lines involving at least one DeLee and Charnley zone were found in $22 \%$ of the surviving hips where the initial post-operative radiograph was available for study. The acetabular component used in this series did not have an outward facing flange at the periphery, a design feature which has been shown to aid pressurisation of cement $^{24,25}$ and to reduce the incidence of radiological demarcation at the cement-bone interface. ${ }^{26}$ Progression of radiolucent lines was seen around only one of these implants. The two acetabular components with radiolucencies seen in all three zones on the initial radiographs have not subsequently migrated and the patients remain asymptomatic. It was reassuring to see that the rate of formation of these lines in two or more zones at final review (11 hips, 10\%) remained low as did the number revised for aseptic loosening of the acetabular component (13 hips).

Limitations of this study include those patients who were unable to return and those with incomplete radiographs after the films had been destroyed by the health authority.
We used the Livermore technique ${ }^{14}$ to measure linear wear. The technique is limited by the variable quality of the radiographs and the fact that measurements are necessarily made in two dimensions. The mean linear wear of polyethylene measured was $0.11 \mathrm{~mm} /$ year $(0.0$ to 0.4$)$ with the majority of the femoral heads having a $26 \mathrm{~mm}$ diameter. In a randomised prospective study comparing a cementless acetabular component with a cemented all-polyethylene component when the same femoral component with a size $26 \mathrm{~mm}$ head, was used, the mean wear rate with cementless component was $0.15 \mathrm{~mm}$ per year compared with $0.07 \mathrm{~mm}$ per year with the cemented design. ${ }^{27}$ The difference in wear was significant $(\mathrm{p}<0.0001)$.

Despite good results achieved with the use of the allpolyethylene concentric Exeter acetabular component this design has been superceded due to the improved radiological findings and survivorship reported with a cemented flanged acetabular component, which allows sustained high cement pressurisation at the time of implantation. ${ }^{26}$

The concentric all-polyethylene Exeter acetabular component and Exeter Universal femoral component show excellent long-term results. When failure is defined as acetabular revision for any reason the survivorship at 15 years, with 61 hips at risk was $89.9 \%$ (95\% CI 84.6 to 95.2) in this series of cemented acetabular components, which is superior to reported series of uncemented implants over a similar period. ${ }^{28-31}$

\section{Listen live \\ Listen to the abstract of this article at www.jbjs.org.uk}

The authors acknowledge the support of the Exeter Hip Research Team in organising patients' appointments, searching for radiographs and providing outcome scores.

The author or one or more of the authors have received or will receive benefits for personal or professional use from a commercial party related directly or indirectly to the subject of this article. In addition, benefits have been or will be directed to a research fund, foundation, educational institution, or other nonprofit organisation with which one or more of the authors are associated.

\section{References}

1. Ritter MA, Keating EM, Faris PM, Brugo G. Metal-backed acetabular cups in total hip arthroplasty. J Bone Joint Surg [Am] 1990;72-A:672-7.

2. Williams HD, Browne G, Gie GA, et al. The Exeter universal cemented femoral component at 8 to 12 years: a study of the first 325 hips. J Bone Joint Surg [Br] 2002;84-B:324-34

3. Carrington NC, Sierra RJ, Gie GA, et al. The Exeter Universal cemented femoral component at 15 to 17 years: an update on the first 325 hips. J Bone Joint Surg $[B r] 2009 ; 91-B: 730-7$.

4. Hardinge K. The direct lateral approach to the hip. J Bone Joint Surg $[\mathrm{Br}]$ 1982;64-B:17-19.

5. Charnley J. Numerical grading of clinical results. In: Low friction arthroplasty of the hip: theory and practice. Berlin: Springer-Verlag, 1979:20-4

6. Harris WH. Traumatic arthritis of the hip after dislocation and acetabular fractures: treatment by mold arthroplasty: an end-result study using a new method of result evaluation. J Bone Joint Surg [Am] 1969;51-A:737-55.

7. Dawson J, Fitzpatrick R, Carr A, Murray DW. Quesionnaire on the perceptions of patiens about total hip replacement. J Bone Joint Surg [Br] 1996;78B:185-90.

8. Murray DW, Fitzpatrick R, Rogers K, et al. The use of the Oxford hip and knee scores. J Bone Joint Surg [Br] 2007;89-B:1010-14

9. Charnley J. The long-term results of low-friction arthroplasty of the hip performed as a primary intervention. J Bone Joint Surg [Br] 1972;54-B:61-76. 
10. Ferdinand RD, Pinder IM. Survival analysis of joint replacements. J Bone Joint Surg [Br] 1997;79-B:878.

11. Kobayashi S, Takaoka K, Saito N, Hisa K. Factors affecting aseptic failure of fixation after primary Charnley total hip arthroplasty: multivariate survival analysis. J Bone Joint Surg [Am] 1997;79-A:1618-27.

12. DeLee JG, Charnley J. Radiological demarcation of cemented sockets in tota hip replacement. Clin Orthop 1976;121:20-32.

13. Gruen TA, McNeice GM, Amstutz HC. "Modes of failure" of cemented stemtype femoral components: a radiographic analysis of loosening. Clin Orthop 1979;141:17-27.

14. Livermore J, Ilstrup D, Morrey B. Effect of femoral head size on wear of the polyethylene acetabular component. J Bone Joint Surg [Am] 1990;72-A:518-28.

15. Murray DW, Carr AJ, Bulstrode C. Survival analysis of joint replacements. J Bone Joint Surg [Br] 1993;75-B:697-704.

16. Dobbs HS. Survivorship of total hip replacements. J Bone Joint Surg $[\mathrm{Br}]$ 1980;62-B:168-73.

17. Hodgkinson JP, Shelley P, Wroblewski BM. The correlation between the roentgenographic appearance and operative findings at the bone-cement junction of the socket in Charnley low friction arthroplasties. Clin Orthop 1988;228:105-9

18. Ackroyd CE, Whitehouse SL, Newman JH, Joslin CC. A comparative study of the medial St Georg sled and kinematic total knee arthroplasties: ten-year survivorship. J Bone Joint Surg [Br] 2002;84-B:667-72.

19. Lettin AW, Ware HS, Morris RW. Survivorship analysis and confidence intervals: an assessment with reference to the Stanmore total knee replacement. $J$ Bone Joint Surg [Br] 1991;73-B:729-31.

20. Bland M. An introduction to medical statistics. Second ed. Oxford: Oxford University Press, 1995.
21. Paprosky WG, Perona PG, Lawrence JM. Acetabular defect classification and surgical reconstruction in revision arthroplasty: a 6-year follow-up evaluation. $J$ Arthroplasty 1994:9:33-44.

22. Kärrholm J, Garellick G, Herberts P. Swedish Hip Arthroplasty Register: annual report 2006. http://www.jru.orthop.gu.se (date last accessed 25 May 2010).

23. No authors listed. Norwegian Hip Arthroplasty Register: annual report 2007. http:/ /www.haukeland.no/nrl/eng (date last accessed 25 May 2010).

24. Oh I, Sander TW, Treharne RW. Total hip acetabular cup flange design and its effect on cement fixation. Clin Orthop 1985;195:304-9.

25. Shelley P, Wroblewski BM. Socket design and cement pressurisation in the Charnley low-friction arthroplasty. J Bone Joint Surg [Br] 1988;70-B:358-63.

26. Hodgkinson JP, Maskell AP, Paul A, Wroblewski BM. Flanged acetabular components in cemented Charnley hip arthroplasty: ten-year follow-up of 350 patients. J Bone Joint Surg [Br] 1993;75-B:464-7.

27. McCombe P, Williams SA. A comparison of polyethylene wear rates between cemented and cementless cups: a prospective, randomised trial. J Bone Joint Surg [Br] 2004;86-B:344-9.

28. Engh CA, Hopper RH Jr, Engh CA Jr. Long-term porous-coated cup survivorship using spikes, screws, and press-fitting for initial fixation. J Arthroplasty 2004;19(Suppl 2):54-60.

29. Parvizi J, Sullivan T, Duffy G, Cabanela ME. Fifteen-year clinical survivorship of Harris-Galante total hip arthroplasty. J Arthroplasty 2004;19:672-7.

30. Della Valle CJ, Berger RA, Shott S, et al. Primary total hip arthroplasty with a porous-coated acetabular component: a concise follow-up of a previous report. $J$ Bone Joint Surg [Br]2004;84-B:1217-22.

31. Gaffey JL, Callaghan JJ, Pedersen DR, et al. Cementless acetabular fixation at fifteen years: a comparison with the same surgeon's results following acetabular fixation with cement. J Bone Joint Surg [Am] 2004;86-A:257-61. 